\title{
The Near-IR [SIII] Lines in a Sample of Star-Forming Galaxies: Chemical Abundances
}

\author{
C. Kehrig ${ }^{1,2}$, J. M. Vílchez ${ }^{1}$, E. Telles ${ }^{2}$, F. Cuisinier ${ }^{3}$, \\ and E. Pérez-Montero ${ }^{4}$ \\ ${ }^{1}$ Instituto de Astrofísica de Andalucía (CSIC) \\ Camino Bajo de Huetor 50, Apartado 3004, 18080 Granada, Spain \\ email: kehrig@iaa.es \\ ${ }^{2}$ Observatório Nacional, Rua General José Cristino 77, Rio de Janeiro, Brazil \\ ${ }^{3}$ GEMAC, Observatório do Valongo/UFRJ,Ladeira do Pedro Antônio 43, Rio de Janeiro, \\ Brazil \\ ${ }^{4}$ Departamento de Física Teórica, C-XI, Universidad Autónoma de Madrid, 28049 Madrid, \\ Spain
}

\begin{abstract}
We present a detailed spectroscopic study of a sample of 34 star-forming dwarf galaxies, ranging from the blue to near-infrared $(\lambda 3700 \AA-1 \mu \mathrm{m})$ (Kehrig et al. 2006). The metal enrichment in this kind of objects has been operating typically at low metallicity enviroments. The spectra were observed with the $1.52 \mathrm{~m}$ telescope at La Silla/ESO. We derive fundamental parameters for HII regions and ionizing sources in our star-forming galaxies, as well as gaseous metal abundances. All the spectra include the nebular[SIII] $\lambda \lambda 9069,9532 \AA$ lines, that are of crucial importance in the derivation of the $\mathrm{S} / \mathrm{H}$ abundances, and relevant ionization diagnostics. We study the relative hardness of their ionizing sources using the $\eta$ ' parameter (Vílchez \& Pagel 1988), and exploring the roles played by metallicity and age. The ionic and total $\mathrm{O} / \mathrm{H}$ was also derived using direct determinations of the $\mathrm{t}_{e}[\mathrm{OIII}]$. The mean $\mathrm{S} / \mathrm{O}$ ratio derived in this work is constant and slightly below the solar $(\mathrm{S} / \mathrm{O})_{\odot}$ value (see fig 1$)$. The data presented here are consistent with the conclusion that $\mathrm{S} / \mathrm{O}$ remains constant as $\mathrm{O} / \mathrm{H}$ varies among the sample of HII galaxies. Variations in $\mathrm{S} / \mathrm{O}$ along the whole $\mathrm{O} / \mathrm{H}$ abundance range may be present, but the scatter in $\mathrm{S} / \mathrm{O}$ (due mainly to observational errors) is still large to constrain them. The assumption that the $\mathrm{S} / \mathrm{O}$ ratio remains constant for all abundances is still an open question and should be explored further (Pérez-Montero et al. 2006).
\end{abstract}

Keywords. ISM: abundances, ISM: HII regions, Galaxies: abundances, Galaxies: dwarf

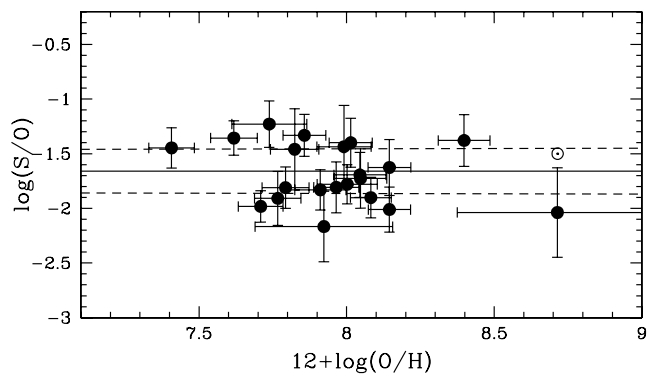

Figure 1. The observed $\mathrm{S} / \mathrm{O}$ abundance ratio for the subset of galaxies of the sample with te [OIII] versus their oxygen abundance. The solar value is shown. The dashed lines are $+/-(1 \sigma)$ of the mean as shown by the continuous line.

\section{References}

Kehrig, C., Vílchez, J.M., Telles, E., Cuisinier, F. \& Pérez-Montero, E. 2006, A\&3A, 457, 477.

Pérez-Montero, E., Díaz, A.I., Vílchez, J.M. \& Kehrig, C. 2006, A\& A , 449, 193.

Vílchez, J.M., \& Pagel, B.E.J. 1988, MNRAS , 231, 257. 